\title{
Undesired Radiation Suppression Technique with Adaptive Control for Distributed Array Antenna Systems in Mobile Environment
}

\author{
Hideya So, Member, IEEE, Kouhei Suzaki, Non-member, and Daisuke Goto, Non-member
}

\begin{abstract}
We have proposed a distributed array antenna (DAA) system for high-speed satellite communications. The DAA system uses multiple small tracking antennas and combines the transmission signals in-phase to increase the antenna gain. DAA system has a problem that the undesired radiation at the sidelobe direction increases as the antenna gain at the main lobe direction increases. In the mobile environment, the conventional technique can suppress the undesired radiation in the limited condition because of changing the direction of the undesired radiation according to the movement of the mobile station. This paper proposes a DAA technique that suppresses the undesired radiation by setting a transmission plate at each antenna aperture and moving them via adaptive control. The transmission plate consisting of a metal patch or slot retransmits electromagnetic waves and changes the amplitude and phase of the waves. To change the radiation patters of each antenna, the transmission plate rotates according to movement of the mobile station. After combining these changed signals, the adaptive control selects the rotate angle of the transmission plate to decrease the undesired radiation at the sidelobe direction. The antenna gain on the main lobe direction after combining is achieved with lower loss because the insertion loss through the transmission plate is smaller. The proposed technique offers more than $2.4 \mathrm{~dB}$ improvement with three antennas and more than $3.5 \mathrm{~dB}$ improvement with four antennas assuming each consists of $8 \times 8$ patches.
\end{abstract}

Index Terms-Distributed array antenna (DAA) system, suppression of undesired radiation, mobile environment, transmission plate, frequency-selective surfaces.

\section{INTRODUCTION}

Greater capacity is being demanded from broadband satellite systems [1]. Satellite communication is especially important over wide ocean areas, which are not supported by landbased terrestrial networks. High-speed satellite communication services are generally realized by using high gain antennas to increase the equivalent isotropically radiated power (EIRP) and the carrier to noise power ratio (CNR). Increasing the antenna aperture can improve the antenna gain, but antenna weight and volume are increased disproportionately, and small vessels have no space for large antennas. We proposed the

Manuscript received April 02, 2020; revised April 29, 2020. Date of publication May 11, 2020. Date of current version May 11, 2020.

H. So and K. Suzaki are with the NTT Network Innovation Laboratories, NTT Corporation, Japan, e-mails: so.hideya@m.ieice.org, suzaki.kouhei@lab.ntt.co.jp.

D. Goto is with the NTT Access Network Service Systems Laboratories, NTT Corporation, Japan, e-mail: goto.daisuke@lab.ntt.co.jp.

Digital Object Identifier (DOI): 10.24138/jcomss.v16i2.1044 distributed array antenna (DAA) system; it combines multiple small antennas to create a larger virtual antenna aperture [2].

A DAA system has multiple small tracking antennas and a DAA controller. DAA system synthesizes transmitted and received signals of the multiple small antennas to establish one large virtual aperture antenna that offers high EIRP and high CNR. DAA system has two other major advantages. First is antenna diversity, which offers improvements in rain margin, and significant redundancy by the use of multiple antennas. Second advantage is the easy installation made possible by the use of small antennas as space is limited on vessels, planes and buildings.

In DAA system, the distance between antennas could be more than one wavelength and multiple grating lobes are generated. Each grating lobe increases the amplitude in the sidelobe direction. Therefore, high antenna gain on the sidelobe directions creates undesired radiation. When another satellite lies in the sidelobe direction, the undesired radiation interferes with the other satellite [3]. It is necessary to reduce the transmission power to reduce interference to give to other satellites. However, the gain of DAA is not achieved when the transmission power decreases. Clearly the undesired radiation in the sidelobe direction must be decreased. Using a low sidelobe antenna is one approach, but the sidelobe value and the antenna size have a trade-off relationship. Since DAA system uses multiple small antennas, low sidelobe antennas are not suitable.

Adaptive arrays and an antenna placement technique have been proposed to suppress the undesired radiation for small antenna array systems [4]-[8]. The adaptive array approach [4]-[6] controls the amplitude and phase of the transmitted signals so as to set nulls on sidelobe directions but prior information as to the sidelobe directions is necessary. Furthermore, suppressing the undesired radiation depresses the antenna gain on the main lobe direction. On the other hand, the antenna placement approach cuts the grating lobes by careful placement of the antennas. One technique uses irregular antenna spacing to disperses the grating lobe [7]. Another technique places the antennas so as to match the null of the array response vectors and the peak of one antenna [8]. Unfortunately, these techniques assume a fixed environment. In mobile environments, the direction of the mobile station to the receiver is continually changing, which renders these techniques ineffective. 

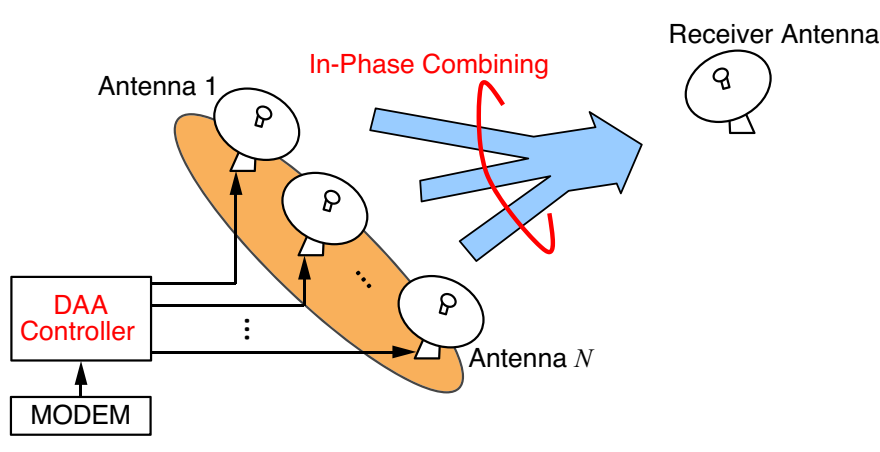

Fig. 1. Concept of DAA system.

This paper proposes the use of adaptively controlled transmission plates to suppress the undesired radiation when DAA system is used in a mobile environment. The plate transmits electromagnetic waves only in a specific band and can control the amplitude and phase of the waves by altering the incident angle of the plate to the waves. The transmission plate is realized as a frequency-selective surface [9]. The transmission plate is set on the aperture of each antenna and rotated so that the radiation pattern of the antenna becomes asymmetrical. The undesired radiation is suppressed by combining the array of asymmetrical patterns. The adaptive control rotates the transmission plate according to the movement of the mobile station. The proposed technique can suppress the undesired radiation with lower loss of the antenna gain on the main lobe direction because of controlling the transmission plate without the antenna placement or the adaptive array.

The rest of this paper is organized as follows. Section II presents DAA system concept and the problems raised by mobile environments. Section III describes the proposed scheme. Computer simulation results are shown in Section IV. Finally, the paper is concluded in Section V.

\section{Distributed Array Antenna (DAA) System}

\section{A. Concept of DAA System}

Fig. 1 shows an application image of DAA system. DAA system consists of multiple small tracking antennas, an DAA controller, and a modem. The DAA controller is placed between the modem and the antennas. At the transmitter side, the DAA controller divides the transmitted signal into $N$ streams and controls the phase and amplitude of each antenna appropriately to realize spatial in-phase combining at receiver antenna. At the receiver side, the DAA controller combines all received signals appropriately to maximize the CNR. DAA system offers both easy installation and significant CNR and EIRP improvements. It also offers inherent redundancy, if one antenna fails, the communication service is supported by the other antennas.

\section{B. Issue of DAA System: Increasing in Undesired Radiation}

DAA system has a problem that the undesired radiation increases when the antenna gain at main lobe increases as the antenna gain at sidelobe increases. $\theta$ is defined as a direction of the receiver. The $n$-th antenna is set to $\left(x_{n}, y_{n}\right) . E_{n}(\theta, \phi)$
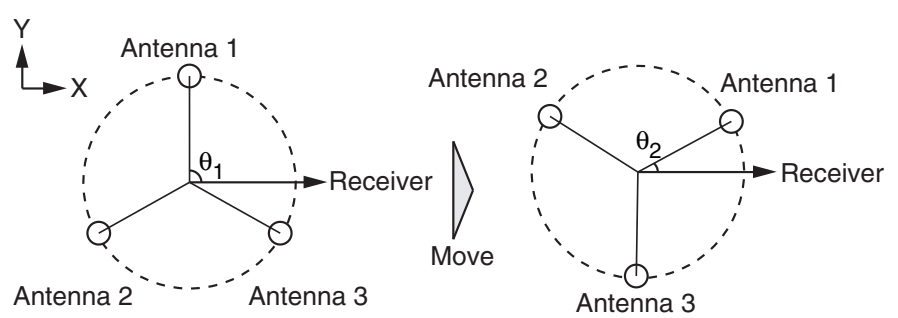

(a) Configuration $1\left(\theta=\theta_{1}\right)$

(b) Configuration $2\left(\theta=\theta_{2}\right)$

Fig. 2. Increasing of undesired radiation by direction angle change of mobile station.

is defined as an array response vector at the $n$-th antenna in azimuth angle, $\phi(-180 \leq \phi \leq 180)$, and can be expressed as

$$
E_{n}(\theta, \phi)=\frac{2 \pi}{\lambda}\left(x_{n} \cos (\theta-\phi)+y_{n} \sin (\theta-\phi)\right) .
$$

$g_{n}(\phi)$ is defined as the radiation pattern of the $n$-th one tracking antenna. The array combining pattern, $G(\theta, \phi)$, is defined as

$$
G(\theta, \phi)=\sum_{n=1}^{N} g_{n}(\phi) E_{n}(\theta, \phi)
$$

The DAA controller controls these vectors to realize inphase combining. When DAA is applied to the fixed station environment, that $\theta$ is static, the optimization of the antenna placement can decrease the undesired radiation [7], [8]. On the other hand, in mobile station environment, the optimization of the antenna placement cannot suppress the undesired radiations because the antenna placement according to the received antenna changes by moving. In other words, $E_{n}(\theta, \phi)$ changes by changing the path length at each antenna when the mobile station moves. In mobile station environment, $\theta$ is re-defined as the mobile station angle.

An example of increasing undesired radiation by changing mobile station environment is shown. In this case, the example has three antennas $(N=3)$ for DAA system. Fig. 2 shows the placement of three antennas. The antenna placement changes by the mobile station moving. Fig. 3 shows the sum of array response vectors $\sum_{n=1}^{N} E_{n}(\theta, \phi)$, the radiation pattern of one tracking antenna, and the array combining radiation pattern of each configuration. In configuration $1\left(\theta=\theta_{1}\right)$, $\sum_{n=1}^{N} E_{n}(\theta, \phi)$ at $\phi=15^{\circ}$ is null, that the radiation pattern of one antenna is the first sidelobe. So the undesired radiation is decreased after array combining in Fig. 3 (c). However, in configuration $2\left(\theta=\theta_{2}\right), \sum_{n=1}^{N} E_{n}(\theta, \phi)$ is not null at $\phi=15^{\circ}$. Note that the relative antenna placement of each antenna is hold. The array combining radiation pattern at $\phi=15^{\circ}$, that is the undesired radiation, increases by changing the antenna placement in configuration 2 . This paper proposes the suppression technique of the undesired radiation in the mobile environment, which add on the existing antenna.

\section{iII. Proposal: Adaptive Control of Transmission PlATE FOR DAA SYSTEM}

Fig. 4 shows the concept of the proposed method. The mobile station has $N$ tracking antennas, $N$ transmission plates, 


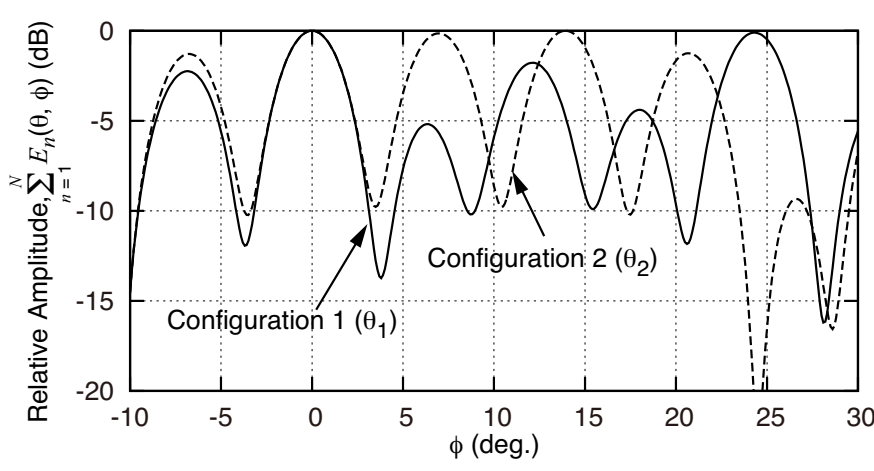

(a) Sum of array response vectors, $\sum_{n=1}^{N} E_{n}(\theta, \phi)$

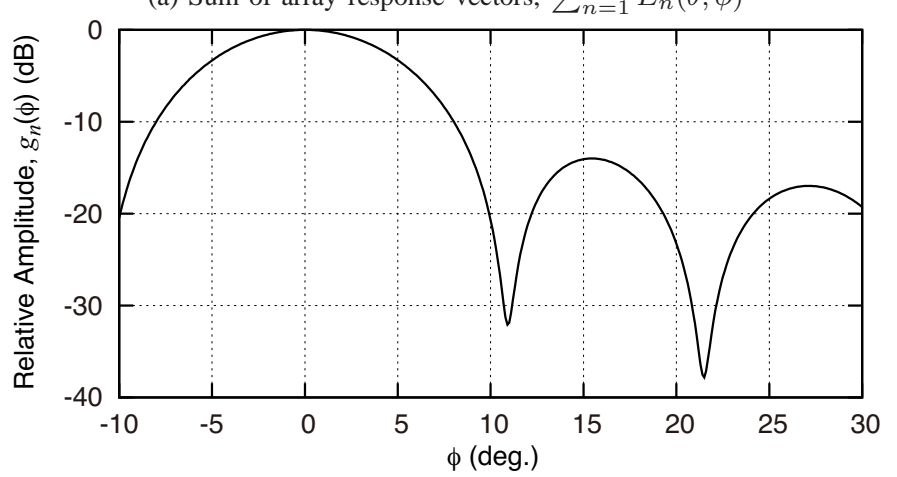

(b) Radiation pattern of one tracking antenna, $g_{n}(\phi)$

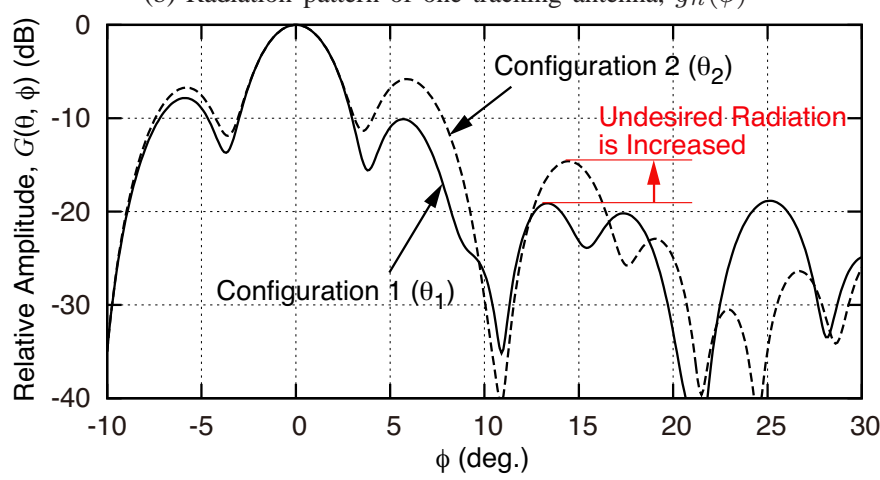

(c) Array combining radiation patterns, $G(\theta, \phi)$

Fig. 3. Increasing of undesired radiation by direction angle change of mobile station.

and a control unit for each transmission plate. The control unit rotates its transmission plate, which is placed in front of the antennas aperture, to suit the mobile stations movements. As the plate we use a frequency-selective surface (FSS) [9] consisting of a periodically structured metal patch or slot. The incident angle of the FSS relative to the aperture, changed by rotating the plate, changes the phase and amplitude of the transmission wave. This changes the radiation pattern of one antenna, $g_{n}(\phi)$. The incident angles of the transmission plates are decided so as to minimize the undesired radiation after array combining.

An example explains a principle of the undesired radiation suppression. Fig. 5 shows the radiation patterns of one antenna with and without the transmission plate. $p_{1}(\phi)$ is the radiation pattern without the transmission plate and $p_{2}(\phi)$ is the radiation pattern with the transmission plate. The first sidelobe

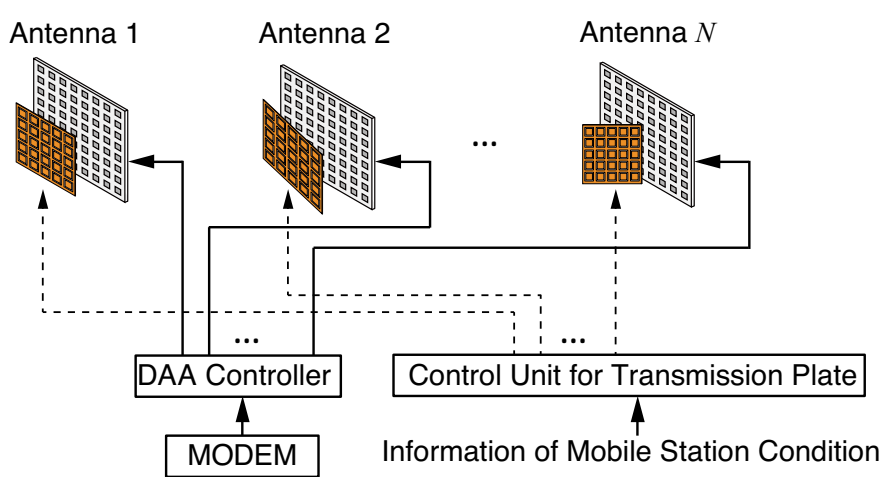

Fig. 4. Concept of suppression technique which uses movable transmission plate.

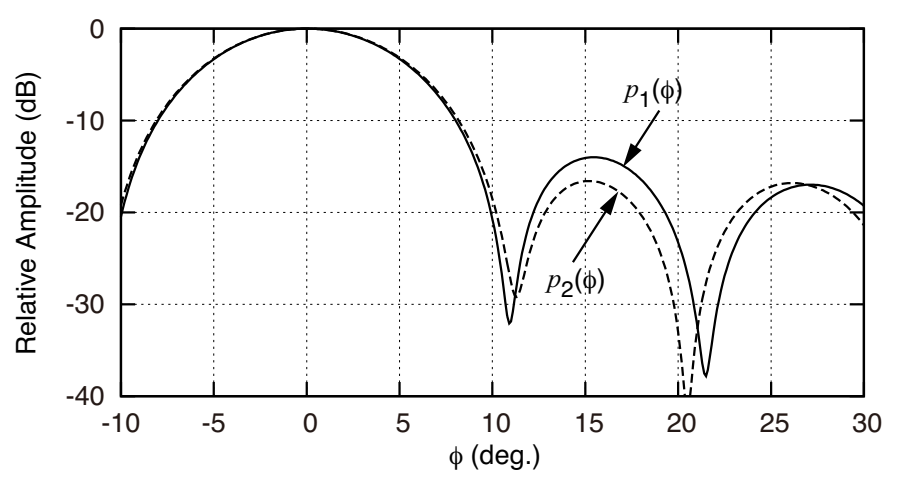

Fig. 5. Radiation patterns of one antenna with and without transmission plate.

of $p_{2}(\phi)$ is decreased than that of $p_{1}(\phi)$. In the configuration 2 (Fig. 2 (b)), the antenna 1 uses $p_{2}(\phi)\left(g_{1}(\phi)=p_{2}(\phi)\right)$ and others uses $p_{1}(\phi)\left(g_{2}(\phi)=g_{3}(\phi)=p_{1}(\phi)\right)$. The array combining radiation pattern is shown in Fig. 6 . The undesired radiation at $\phi=15^{\circ}$ is decreased. The control unit chooses the most suitable radiation pattern every antennas according to the mobile station angle $\theta$.

There is a table that expresses the relationship between the undesired radiation after combining and the rotation angle of each transmission plate in advance. The proposed technique selects the rotation angles from the table according to the mobile stations relative position. Note that the proposed technique can be combined with the conventional techniques shown in

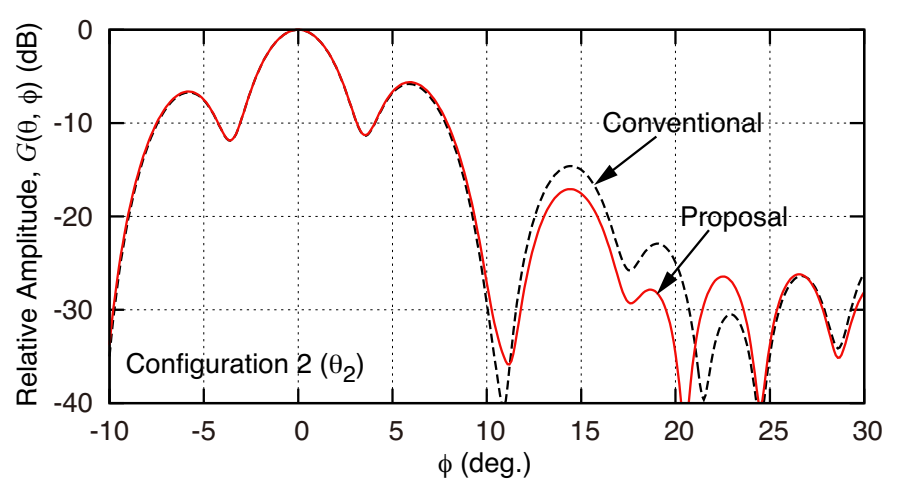

Fig. 6. Array combining radiation patterns. 


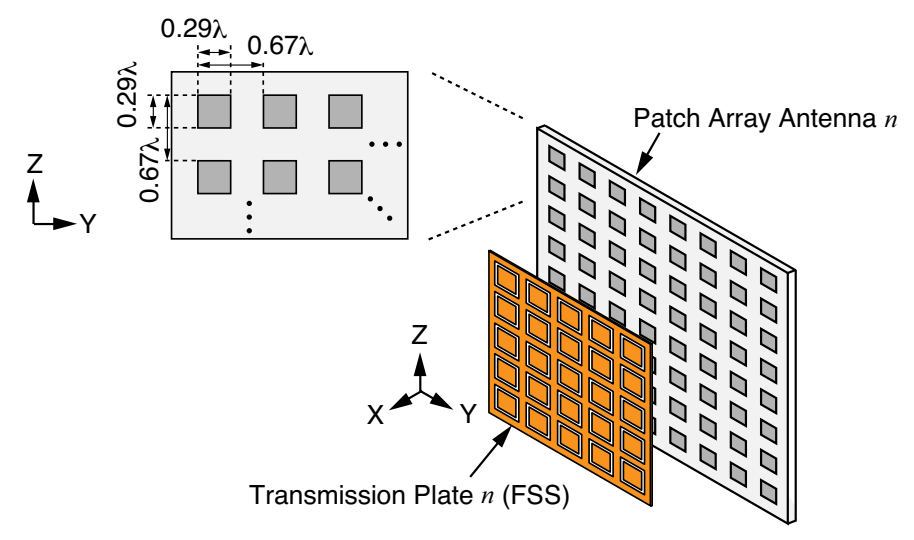

(a) Perspective view

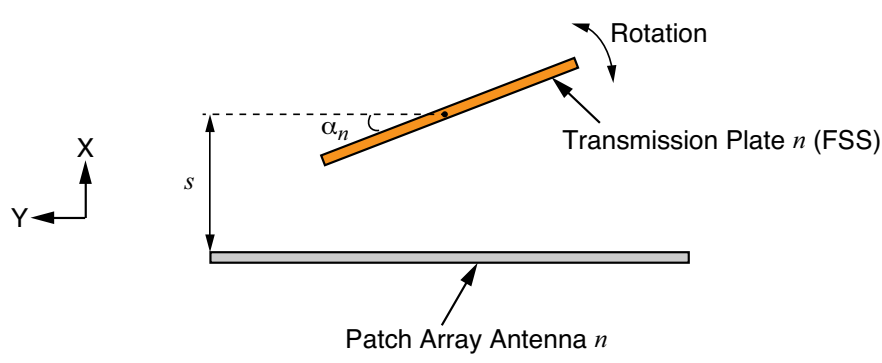

(b) Top view

Fig. 7. $8 \times 8$ patch array antenna configuration employing transmission plate.

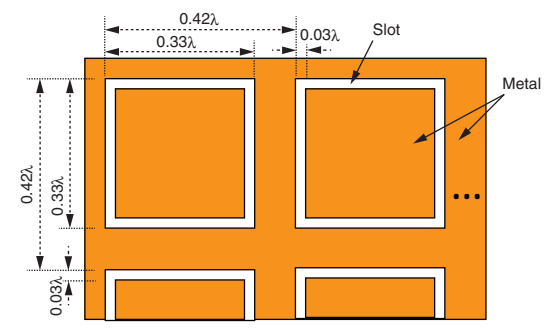

Fig. 8. Transmission plate.

[4]-[7].

\section{Performance Evaluation}

Simulations were conducted to verify the effectiveness of the proposal. First, an electromagnetic field analysis was performed to calculate the radiation patterns of one antenna with the transmission plate. Next, the array combining radiation patterns were simulated using the array response vector of each antenna and the radiation patterns calculated in a preliminary step [11]. DAA system employs the patch array antenna as the one antenna because the transmission plate can be placed near the aperture of the antenna. In this paper, DAA system uses three or four antennas, which are the $8 \times 8$ patch array antennas.

\section{A. One Antenna Design}

Fig. 7 shows a $8 \times 8$ patch array antenna with movable transmission plate. The patch array antenna consists of 64 patches, see Fig. 7 (a) for details. $\lambda$ is a wavelength of the resonant frequency. The patch array antenna is made on a

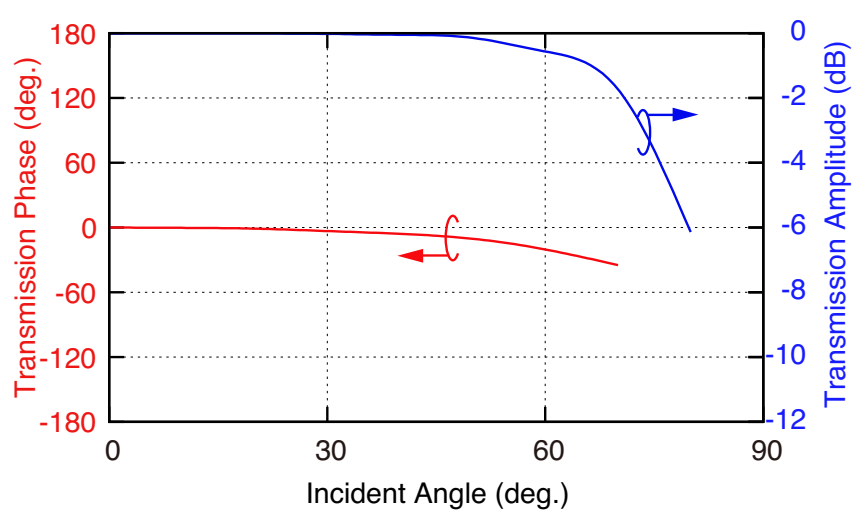

Fig. 9. Transmission characteristics of transmission plate.

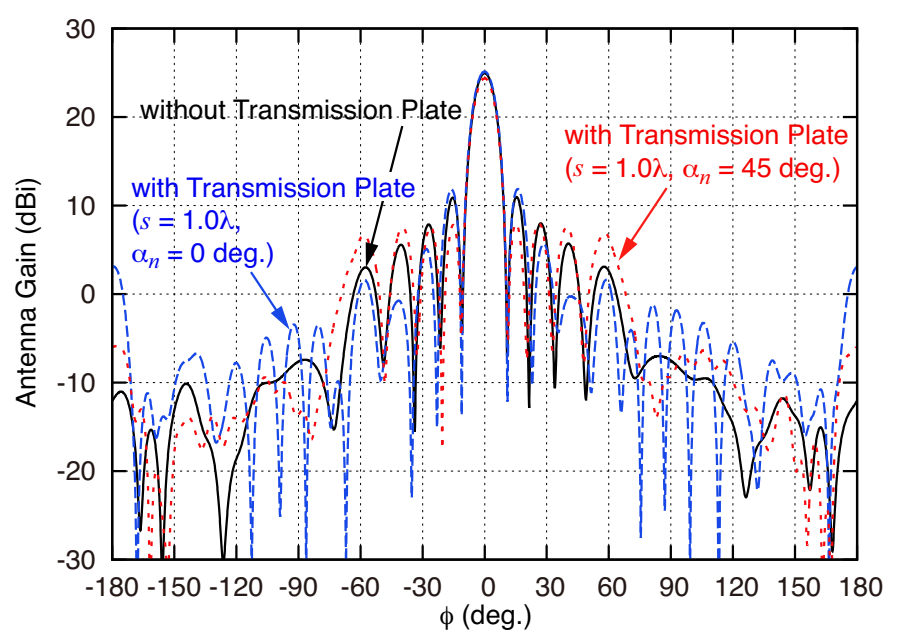

Fig. 10. Radiation pattern of one antenna with and without transmission plate in the horizontal plane.

print circuit board, and that relative permittivity is 2.6 . The array is fed from the backside of the antenna element. The transmission plate and the patch array antenna lie on the same X-axis. The distance between the transmission plate and the patch array antenna is defined as $s$. The transmission plate rotates around the $\mathrm{Z}$-axis at the center of the plate as shown in Fig. 7 (b), and changes the radiation pattern in the horizontal plane. The rotation angle of $n$-th transmission plate is defined as $\alpha_{n}$. The rotation control unit is not shown in Figs for simplicity. Details of the transmission plate, which is the $5 \times 5$ slots, are shown in Fig. 8. Fig. 9 shows the amplitude and phase characteristics of the transmission plate versus the incident angle. It can be seen that the insertion loss through the transmission plate is negligible in the front direction. However, the insertion loss increase and the phase changes as the incident angle is increased.

Fig. 10 shows the radiation patterns of one antenna with and without the transmission plate. $s$ was set to $1.0 \lambda$, and $\alpha_{n}$ was set to $0^{\circ}$ and $45^{\circ}$ when the antenna has the transmission plate. Each VSWR is less than 1.5. The peak gain of the radiation pattern with the transmission plate at $\alpha_{n}=0^{\circ}$ is equal as that of the radiation pattern without the transmission plate. The insertion loss of the transmission plate is negligible because 
of $\alpha_{n}=0^{\circ}$. The transmission plate at $\alpha_{n}=0^{\circ}$ changes the sidelobe from that of the antenna without the transmission plate. On the other hand, the peak gain decreases $0.5 \mathrm{~dB}$ when the transmission plate rotated, $\alpha_{n}=45^{\circ}$ because the transmission plate has loss for electromagnetic waves by the diagonal incidence.

Fig. 11 shows the relationship between the power of each sidelobe and $\alpha_{n} . s$ also was set to $1.0 \lambda$. The horizontal axis is the index of each sidelobe starting from the lobe immediately adjacent to the main lobe. The transmission plate changes the asymmetrical pattern by $\alpha_{n}$. Fig. 12 plots the power of the first sidelobe as a function of $s$ when $\alpha_{n}$ is $45^{\circ} . \alpha_{n}$ cannot be set more than $45^{\circ}$ when $s$ is less than $1.0 \lambda$ because the transmission plate touches the antenna element. Therefore, $s$ values less than $1.0 \lambda$, are excluded. The power of the sidelobe increases as $s$ increases because the transmission plate scatters the electromagnetic waves. In this paper, $s$ is set to $1.0 \lambda$ so that the power of the first sidelobe is minimized.

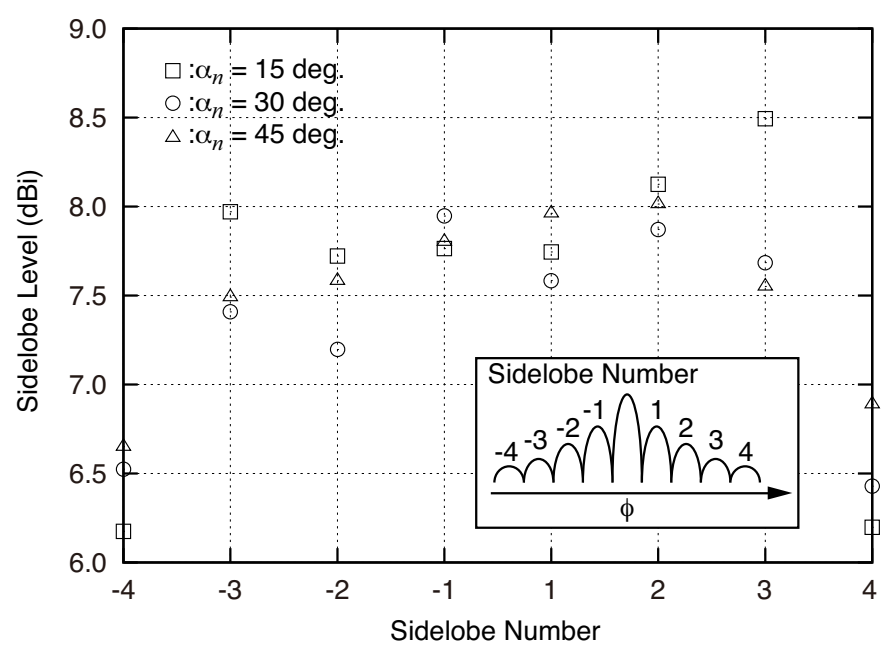

Fig. 11. Relationship between the sidelobe level and rotary angle, $\alpha_{n}$.

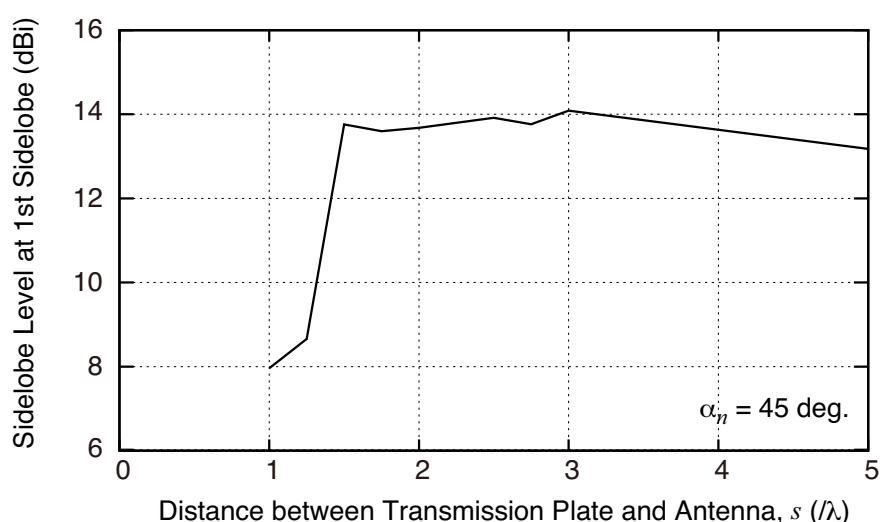

Fig. 12. First sidelobe level as a function of the distance, $s$, when $\alpha_{n}$ is $45^{\circ}$

\section{B. Suppression Effect of Undesired Radiation at Array Com- bining}

Fig. 13 shows the antenna placement with three and four antennas. These antennas are set at equal distances on the circumference with radius of $r$. Each antenna is the same as that detailed in the previous section. Seven plate rotation angles were examined by changing $\alpha_{n}$ from $-45^{\circ}$ to $45^{\circ}$ in steps of $15^{\circ}$. It is assumed that the mobile station has a receiver antenna with $\theta=0^{\circ} \cdot \alpha_{n}$ at each antenna is chosen from the all combinations so that the undesired radiation is minimized. For simplicity, the elevation angle was kept constant at $0^{\circ}$.

The suppression value, $G_{U}(\theta)$, is defined as the difference between the peak gain at $\phi=0^{\circ}$ and the value that is maximum except the main lobe, $10^{\circ} \leq|\phi| \leq 180^{\circ}$. $G_{U}(\theta)$ is shown below,

$$
G_{U}(\theta)=G(\theta, 0)-\max _{10^{\circ} \leq|\phi| \leq 180^{\circ}} G(\theta, \phi) .
$$

The undesired radiation is small so that $G_{U}(\theta)$ is large.
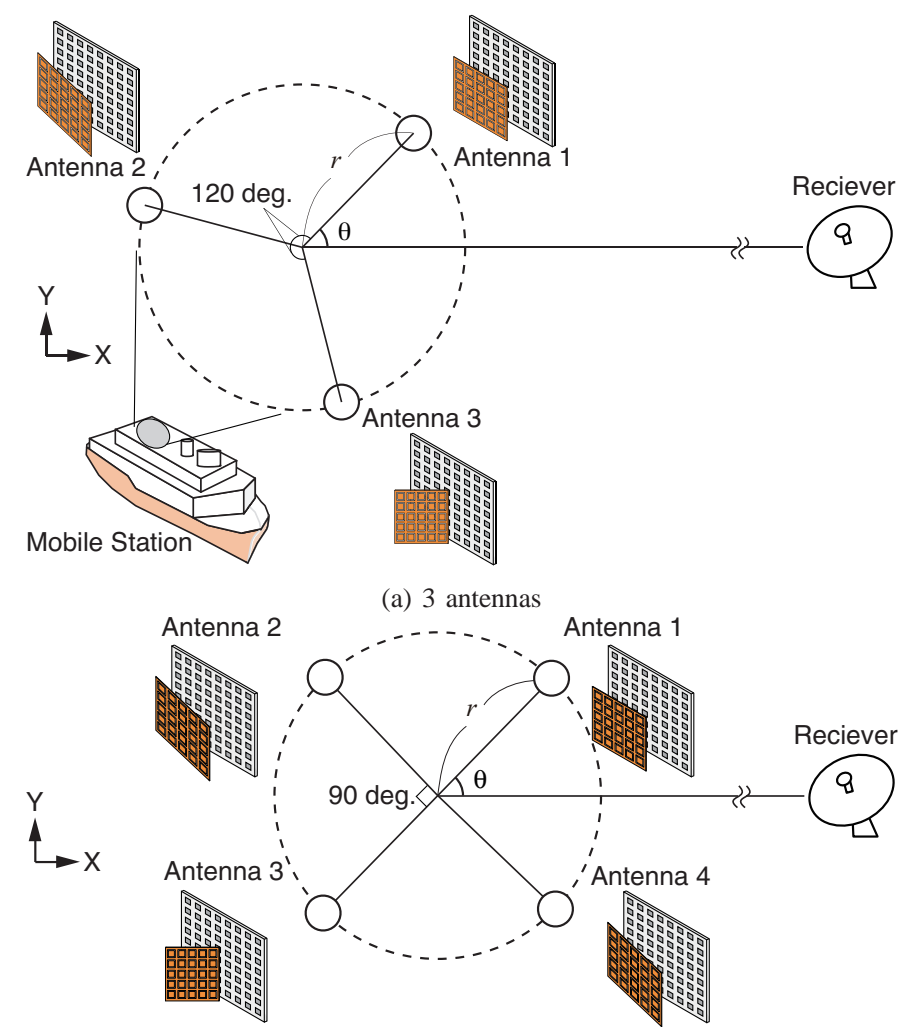

(b) 4 antennas

Fig. 13. Antenna placement.

Fig. 14 shows an array combining radiation pattern of three antennas. For comparison, the performance of the conventional approach, which has no transmission plate and no adaptive control, is shown. The radius of antenna placement, $r$, is assumed to be $5.5 \lambda .5 .5 \lambda$ is almost the smallest placement distance because the antenna size is approximately $5.2 \lambda$. The mobile station angle, $\theta$, is set to $37^{\circ}$ when $G_{U}(\theta)$ of the conventional is worst case. The peak gain is decreased $0.5 \mathrm{~dB}$ by the adaptive control of the transmission plates. However, the undesired radiation at $\phi=15^{\circ}$ is decreased $3.2 \mathrm{~dB}$. The proposal offers an improvement of $2.7 \mathrm{~dB}$ in consideration of the gain loss.

Fig. 15 (a) shows $G_{U}(\theta)$ of three antennas as a function of mobile station angle, $\theta$. $G_{U}(\theta)$ exhibits periodicity because 


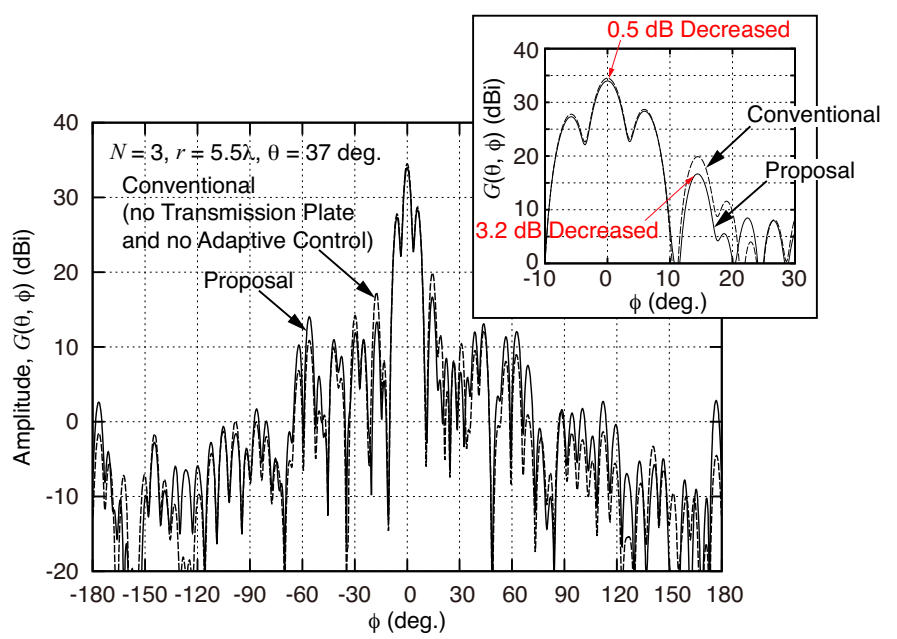

Fig. 14. Comparison of array combining radiation pattern when $G_{U}(\theta)$ of conventional is worst case.

of the circumferential antenna placement. Fig. 15 (b) shows the characteristics for one period; $\theta$ ranges from $0^{\circ}$ to $60^{\circ}$. The maximum transmission power is decided by the maximum interference that the transmitter imposes on the other receiver. Therefore, suppression performance is evaluated from the minimum $G_{U}(\theta)$ that is the worst case. The proposed technique improves the undesired radiation regardless of $\theta$ and offers an improvement of $2.4 \mathrm{~dB}$. The radius of the antenna placement, $r$, is changed. Fig. 16 shows $G_{U}(\theta)$ with three antennas as a function of $\theta$ when $r$ is $10 \lambda$. The proposed technique can suppress the undesired radiation regardless of $\theta$. The minimum $G_{U}(\theta)$ of the proposal is improved $2.9 \mathrm{~dB}$ than that of the conventional.

Finally, the impact of the number of antennas was confirmed. Fig. 17 plots the minimum $G_{U}(\theta)$ for each antenna number as a function of $r$. The proposed technique of each antenna number suppresses the undesired radiation compared to the conventional approach for all antenna numbers examined. The proposed technique with four antennas offers an improvement $3.6 \mathrm{~dB}$ when $r$ is $10 \lambda$, and the improvement is bigger than that with three antennas. Each minimum $G_{U}(\theta)$ of the proposed technique has the same characteristics without dependence on the number of antennas because the optimal value is chosen in $\alpha_{n}$ every antenna.

\section{Conclusions}

This paper has proposed an undesired radiation suppression technique for DAA systems in mobile environments. The proposed technique sets a movable transmission plate at each antenna aperture and controls plate angle to suit the mobile stations relative position. DAA system with an $8 \times 8$ patch array antenna as one antenna was assumed. These antennas were set at equal distances on the circumference. The proposal was found to offer more than $2.4 \mathrm{~dB}$ greater suppression with three antennas and more than $3.6 \mathrm{~dB}$ suppression with four antennas when the radius of the antenna placement was $10 \lambda$. The proposal had no characteristics change by the number of antennas. The rotary angle design, which does not choose from all combinations, is the subject for future study.

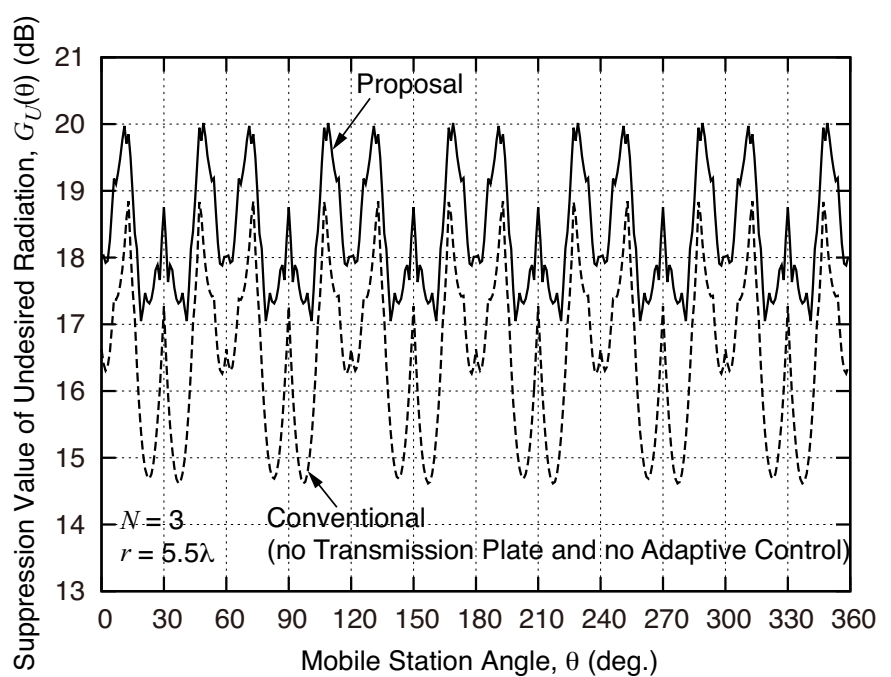

(a) $0^{\circ} \leq \theta \leq 360^{\circ}$

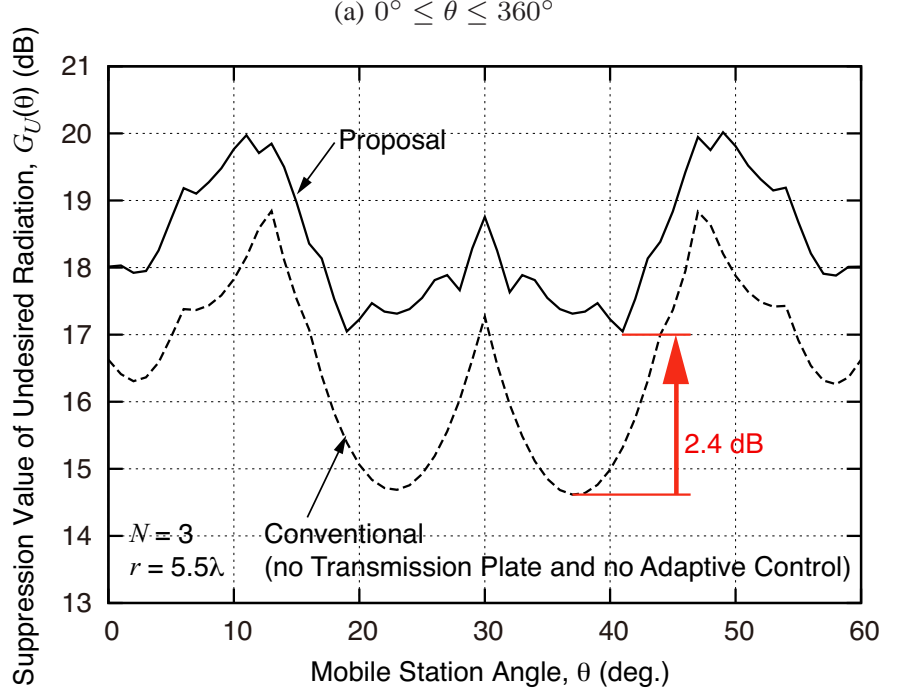

(b) $0^{\circ} \leq \theta \leq 60^{\circ}$

Fig. 15. Undesired radiation value as a function of mobile station angle, $\theta$, with 3 antennas when $r$ is $5.5 \lambda$.

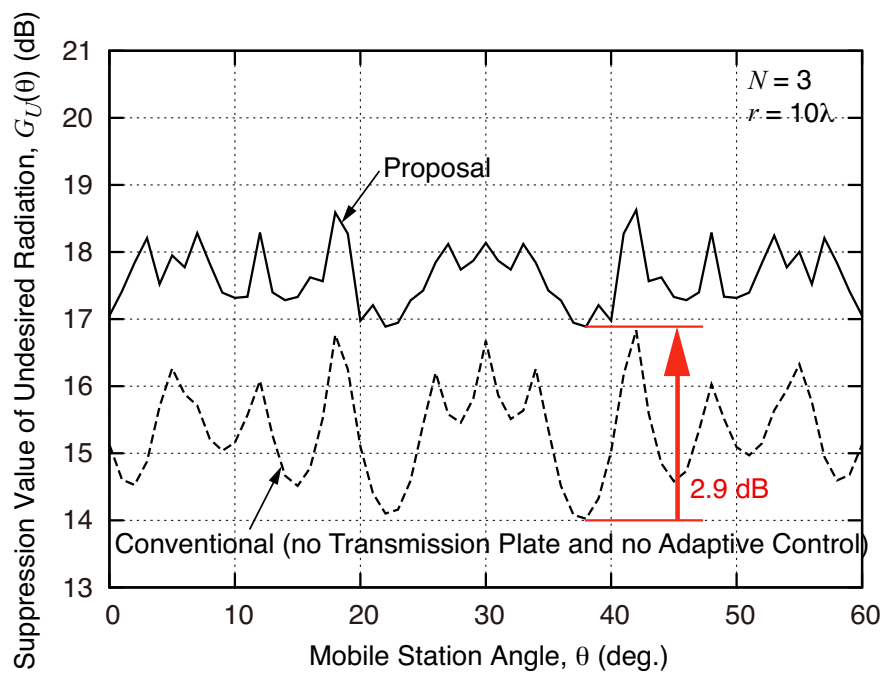

Fig. 16. Undesired radiation change as function of mobile station angle, $\theta$, with 3 antennas when $r$ is $10 \lambda$. 


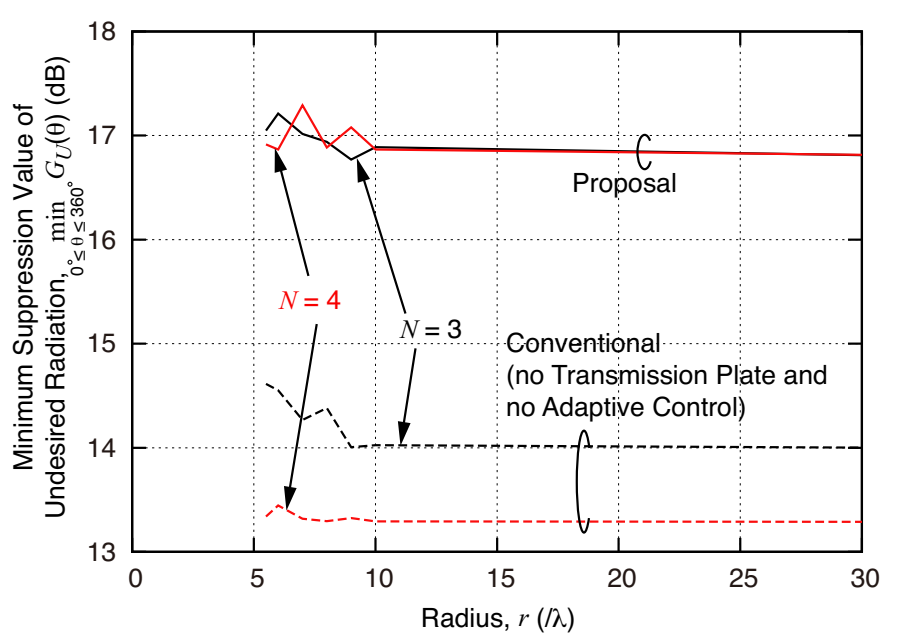

Fig. 17. Minimum of undesired radiation as a function of $r$.

\section{REFERENCES}

[1] O. Vidal, G. Verelst, J. Lacan, E. Alberty, J. Radzik, and M. Bousquet, "Next generation high throughput satellite system," 2012 IEEE First AESS European Conf., pp. 1-7, Oct. 2012. doi: 10.1109/ESTEL.2012.6400146

[2] K. Suzaki, Y. Suzuki, and K. Kobayashi, "A novel earth station antenna concept for Ku-band mobile satellite communication systems Distributed array antenna and key technologies-," 29th AIAA ICSSC2011, Nov.-Dec. 2011. doi: 10.2514/6.2011-8051

[3] F. Yamashita, Y. Suzuki, D. Goto, K. Suzaki, H. So, and K. Kobayashi, "Research and development of technologies on distributed array antenna systems," in Proc. IEICE Conf. 2015, B-3-15, Sept. 2015.

[4] P. W. Howells, "Intermediate frequency sidelobe canceller," U. S. Patent, no. 3202990, Aug. 1965.

[5] R. T. Compton, "Wideband interference cancellation in adaptive sidelobe cancellers," IEEE Trans. on Aerospace and Electronic Systems, vol. AES-19, no. 6, Nov. 1983. doi: 10.1109/TAES.1983.309403

6] M. M. Khodier and C. G. Christodoulou, "Linear array geometry synthesis with minimum sidelobe level and null control using particle swarm optimization," IEEE trans. Antennas Propag., vol. 53, no. 8, pp. 2674-2679, Aug. 2005. doi: 10.1109/TAP.2005.851762

[7] O. Mizokami, T. Nakazawa, and M. Shinriki, "A Study on an arrangement spaces for a non-uniform array in suppressing gratinglobe," IEICE Trans. Commun., vol. J83-B, no.1, pp.141-143, Jan. 2000.

[8] D. Goto, F. Yamashita, K. Suzaki, H. So, and K. Kobayashi, "A proposal on the offset antenna arrangement technique to improve transmitting EIRP in the distributed array antenna systems," in Proc. IEICE Gen. Conf. 2015, B-1-220, March 2015.

[9] B. A. Munk, Frequency selective surfaces: theory and design, WileyInterscience Publication, 2000.

[10] ANSYS HFSS, http://www.ansys.com/.

[11] D. Goto, F. Yamashita, K. Suzaki, H. So, Y. Suzuki, and K. Kobayashi, "Experimental validation of a practical method of estimating distributed array antenna Patterns," JC-SAT 2015, Oct. 2015.

[12] J. D. Kraus, Antennas third edition, Mc Graw Hill, 2002.

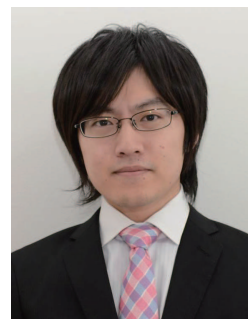

Hideya So received the B.E. degree from Tokyo University of Science, Japan, in 2009, and the M.E. degree from Tokyo Institute of Technology, Japan, in 2011, respectively. He joined NTT Access Network Service Systems Laboratories, NTT Corporation, in 2011. He engaged in has researched on highreliability radio access, base station antennas, and adaptive arrays for the future wireless access systems. He currently works for NTT West Corporation. He received the IEICE Radio Communication Systems (RCS) Active Researcher Award in 2011, the IEICE Young Researcher's Award in 2016, and the IEICE Best Paper Award in 2016. He is a member of the IEICE and IEEE.

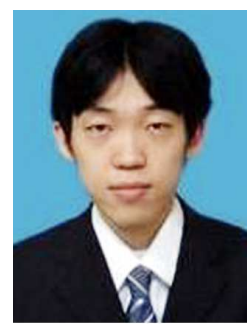

Kouhei Suzaki received the B.E and M.E degree in electrical engineering from Tokyo University of Science, Tokyo, Japan in 2007 and 2009, respectively. In 2009, he joined NTT Access Network Service Systems Laboratories. Since then he has been engaged in research on signal processing of adaptive array antenna and the Satellite Earth Station. He is a member of IEICE.

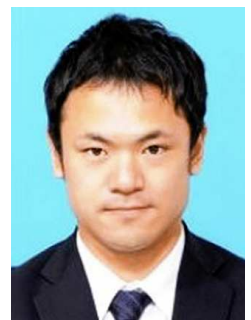

Daisuke Goto received the B.E. degree in system engineering from Shizuoka University and the M.E. degree in electrical engineering and computer science from Nagoya University, Japan, in 2010 and 2012, respectively. In 2012, he joined NTT Access Network Service Systems Laboratories, Japan. His current research interest is satellite communication techniques 